OPEN ACCESS

Edited by:

Alexandre Heeren,

Catholic University of Louvain, Belgium

Reviewed by: Maria Semkovska, University of Southern Denmark Denmark

Grazia Rutigliano, University of Pisa, Italy

${ }^{*}$ Correspondence: Paola Rocca paola.rocca@unito.it

Specialty section: This article was submitted to

Psychopathology,

a section of the journal

Frontiers in Psychiatry

Received: 05 April 2019 Accepted: 06 March 2020 Published: 24 March 2020

Citation:

Montemagni C, Bellino S, Bracale N, Bozzatello P and Rocca P (2020)

Models Predicting Psychosis in Patients With High Clinical Risk: A Systematic Review.

Front. Psychiatry 11:223. doi: 10.3389/fpsyt.2020.00223

\section{Models Predicting Psychosis in Patients With High Clinical Risk: A Systematic Review}

\author{
Cristiana Montemagni, Silvio Bellino, Nadja Bracale, Paola Bozzatello and Paola Rocca* \\ Department of Neuroscience, School of Medicine, University of Turin, Turin, Italy
}

Objective: The present study reviews predictive models used to improve prediction of psychosis onset in individuals at clinical high risk for psychosis $(\mathrm{CHR})$, using clinical, biological, neurocognitive, environmental, and combinations of predictors.

Methods: A systematic literature search on PubMed was carried out (from 1998 through 2019) to find all studies that developed or validated a model predicting the transition to psychosis in CHR subjects.

Results: We found 1,406 records. Thirty-eight of them met the inclusion criteria; 11 studies using clinical predictive models, seven studies using biological models, five studies using neurocognitive models, five studies using environmental models, and 18 studies using combinations of predictive models across different domains. While the highest positive predictive value (PPV) in clinical, biological, neurocognitive, and combined predictive models were relatively high (all above 83 ), the highest PPV across environmental predictive models was modest (63\%). Moreover, none of the combined models showed a superiority when compared with more parsimonious models (using only neurocognitive, clinical, biological, or environmental factors).

Conclusions: The use of predictive models may allow high prognostic accuracy for psychosis prediction in CHR individuals. However, only ten studies had performed an internal validation of their models. Among the models with the highest PPVs, only the biological and neurocognitive but not the combined models underwent validation. Further validation of predicted models is needed to ensure external validity.

Keywords: clinical high risk for psychosis (CHR), attenuated psychotic symptoms (APS), brief and limited intermittent psychotic symptoms (BLIPS), genetic risk and deterioration syndrome (GRD), predictive model

\section{INTRODUCTION}

Psychotic disorders are some of the most serious mental disorders considering the individual and the social impact $(1,2)$. They represented the 11th cause of disability in the world in 2013 (3). The delay between the diagnosis and the treatment ranges from 1 to 3 years (4) and results in worsening clinical outcomes (5). Therefore, the clinical focus has increasingly shifted to the early detection and treatment with the aim to either attenuate, postpone and globally avoid the transition to psychosis (6), or enhance clinical and functional outcomes of psychosis over time $(7,8)$. Psychosis does not 
appear directly in its full-blown form in adults but it gradually develops over time: often the first manifestations already take place in adolescents $(9,10)$. For most of the patients suffering from schizophrenia and psychotic disorders, the onset of the disease is anticipated by different symptoms: slight changes in belief, thought, and perception that represent mild forms of delusions, formal thought disorder, and hallucinations, respectively (11).

The clinical staging model has been created to catch these progressive changes, with progressively increasing levels of severity over time $(12,13)$. This model describes psychopathology in a continuum of different subsequent stages. It comprises five different stages, from stage 0 to stage 4 , starting from the lowest level of increased risk of mental illness to progressively higher stages of severity, leading to separated but overlapping pathologies at the highest levels $(14,15)$. Stage 0 includes subjects at increased risk without any kind of symptoms; stage 1 refers to individuals at clinical high risk for psychosis (CHR); stage 2 coincides with the acute phase or crisis, featured by full-blown psychotic symptoms (the full-threshold first episode psychosis), after which an early recovery phase or post- acute phase in the 6-12 months after the onset of the disease occurs; stage 3 encompasses individuals with either persistent illness or recurrent episodes after the first one (12, $13,16)$ and stage 4 holds subjects with chronic disease.

This psychopathological model allows to stage this pathology so that different types of interventions, depending on the stage of illness, can be developed. The psychopathology would be more susceptible to intervention strategies in the first phases of the disease and more crystallized and resistant to therapies in the last phases (15).

The CHR criteria include: attenuated psychotic symptoms (APS), representing mild positive symptoms; brief and limited intermittent psychotic symptoms (BLIPS), characterized by transient, non-serious psychotic symptoms lasting part of the day, and lasted for a maximum period of one week after which spontaneously went to remission; and genetic risk and deterioration syndrome (GRD), including patients with family history of psychosis or schizotypal personality disorder, with additional decline in functioning (17).

Frequently, research in the area of psychiatry has as principal focus the transition from CHR to First Episode Psychosis. Helpseeking subjects meeting CHR criteria, regardless of the scale used, have an increased risk to develop psychotic disorders (18), within a period of time that can be considered quite short. According to a meta-analysis storing data from 27 studies including a number of 2,502 patients, $18 \%$ of them developed First Episode Psychosis at by 6 months, 22\% by 1 year, 29\% by 2 years, and $36 \%$ by 3 years from initial assessment (19), with about $73 \%$ of these developing a schizophrenia spectrum disorder (20).

Overall, compared to the general population, CHR subjects have a 2-year relative risk (RR) to develop psychosis of $460 \%$ as compared to general population (29\%/0.063\%) (21). However, extracting from the overall high-risk entity, its three principal subgroups, patients with BLIPS were at greater risk for developing psychosis (39\% vs 19\% after 24 months), than patients in the APS and GRD subgroups (22), while the GRD subgroup shows only a slight transition risks of $5 \%$ after three years of follow-up (22).

Since most of the studies conducted a follow-up period of not more than 3 years, after this period the transition rate to psychosis is not completely clear. However, most conversions occur during the first year following the evaluation and the conversion rate decreases significantly thereafter, suggesting that the CHR criteria are sensitive to an imminent risk of the onset of full psychosis (23). However, the CHR criteria alone seem to be insufficient in predicting the imminence of the first episode psychosis, given that from $2 / 3$ to $4 / 5$ cases identified through these instruments do not turn into psychosis within a period of 2 years (24). Thus, the aim is to propose a prognostic model that more effectively picks out those individuals who are more likely to switch from ultra-high risk to a first-episode psychosis (FEP) within a given period of time, to adapt treatments to what subjects really need.

Nevertheless, there is not a model of prediction of the transition to psychosis that has been utilized in clinical practice. One explanation can be that psychotic disorders are heterogeneous in phenomenology, pathophysiology, and etiology (25): it means that CHR samples are composed of different and largely heterogeneous subgroups (26). Another reason can be found in the poor quality of the statistical methods used in the studies involved in developing a transition model from CHR stage to full-blown psychosis. A recent review on 91 studies highlighted several shortcomings of this kind of research: poor methods and reporting, no internal or external cross-validation, small sample sizes, and strategies to create these models not well done. Therefore, most of these models probably have overoptimistic and not realistic predictive accuracy (27).

The present study reviews models predicting transition to psychosis, developed to enhance prediction of illness onset in CHR subjects, extending results of a previous study of prognostic accuracy parameters of predictive modeling studies using clinical, biological, neurocognitive, environmental, and combinations of predictors (28).

\section{METHODS}

\section{Literature Search}

On January 31, 2019, an electronic search on PubMed was carried out (from 1998 through 2019), using the following search terms: "at risk mental state," "psychosis risk," "prodrome," "prodromal psychosis," "high risk," "clinical high risk psychosis", "attenuated psychotic symptoms", "APS”, "brief and limited intermittent psychotic symptoms", "BLIPS", "brief intermittent psychosis syndrome", "BIPS", "genetic risk and deterioration syndrome", "GRD”, "psychosis prediction," "psychosis onset," "predictive model". The research was restricted to those articles published from 1998 onward, 
because this is the year in which the first prospective studies with subjects meeting validated CHR criteria have been published (29).

This qualitative review was executed according to the Preferred Reporting Items for Systematic Reviews and MetaAnalyses (PRISMA) standard, including evaluation of bias (confounding, overlapping data, publication bias) (30). (Figure 1).

Studies were selected in a two-step procedure. First of all, all references retrieved from the databases were screened based on their titles and abstracts. Subsequently, the articles that were potentially eligible were further evaluated based on their full texts. All references within the included studies and those of any previous pertinent reviews were carefully reviewed to identify additional relevant studies. Discrepancies were resolved by mutual discussions. Consensus was then obtained, resulting in a final set of articles that have been reviewed and summarized.

\section{Inclusion/Exclusion Criteria}

As depicted in Figure 1, these are the inclusion criteria for the studies in the present review: (a) original articles, to be published in English; (b) presence of CHR subjects [i.e., APS or GRD or BLIPS or brief intermittent psychosis syndrome (BIPS)] according to international standard criteria (6); (c) inclusion of clinical, biological, neurocognitive, environmental, or combinations of predictors to separate $\mathrm{CHR}$ individuals who later developed psychosis from those who did not; (d) inclusion of rigorous predictive models, algorithms, or learning systems that predicted later transition to psychosis from variables obtained at baseline, like regression (logistic, Cox proportional hazard model, least absolute shrinkage, and selection operator), support vector machines, or greedy algorithms (31-34).

The following were the exclusion criteria: (a) abstracts, pilot datasets, reviews, articles not written in English; (b) not rigorous statistic methods (i.e., use of mean differences or chi square tests); (c) articles with overlapping datasets using the same

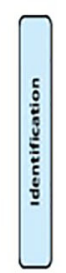

Articles identified through database searching $(n=1327)$
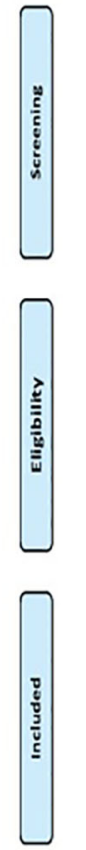

Articles identified

through manual search

$(n=79)$

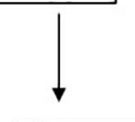

Abstract screened

( $n=1406)$

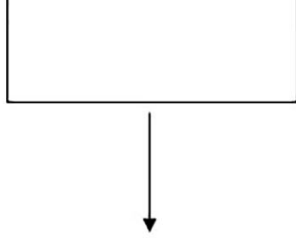

Full-text articles assessed for eligibility ( $n=115)$

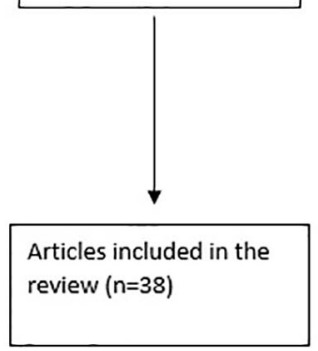

Abstract excluded on initial review $(n=1291)$

FIGURE 1 | PRISMA flow chart. 
predictor. Particularly, when several articles were published using the same population sample, we have chosen the studies reporting the largest sample and most recent data set.

\section{Recorded Variables}

Two investigators (CM, NB) independently realized the extrapolation of data. Several variables have been extracted from the evaluated articles: author, year of publication, demographic characteristics of the CHR sample, predictor domain (clinical, biological, neurocognitive, environmental, combinations), cut-off of predictive variables, type of validation, diagnostic instrument used to define CHR group, administration of antipsychotics, follow-up time, predictive model, and prognostic accuracy data (sensibility SE, specificity SP, positive predictive value PPV, negative predictive value NPV). Moreover, we checked the missing data with all the corresponding authors to record all the information we needed.

\section{RESULTS}

\section{Selection of Studies Search}

Figure 1 describes the details of what has been searched in literature and the reasons why some articles were excluded. The electronic and manual search described in the previous section provided 1,406 records.

Thirty-eight of these studies met the inclusion criteria: 11 studies made use of clinical predictive models, seven studies used biological models, five studies made use of neurocognitive models, five studies used environmental models, and 18 studies made use of combinations of predictive models across different domains. The results are schematically described in Table $\mathbf{1 .}$

For all these studies, validation was evaluated. Some models have internal validation, that means test model in new data, random from underlying population. Other studies have external validation, that means test model in new data, different from development population. Some models have apparent validation, that gives an optimistic estimate of model performance. Some studies have a cross-validation: it means to test the model's ability to predict new data that was not used in estimating it, in order to flag problems like overfitting or selection bias (6) and to give an insight on how the model will generalize to an independent dataset. However, some models do not show any validation.

\section{Clinical Predictive Models}

The 11 studies that have tested the clinical predictive models are described in Tables $\mathbf{1}$ and $\mathbf{2}$. The clinical parameters included specific positive [odd belief: (35); auditory hallucinations: (35, 45); unusual thought content: (36); illogical thinking: (39); suspiciousness: (36, 42, 43); bizarre thinking: (44); delusions: (45); formal thought disorders: (45); disorganized communication: (40,41); positive symptoms: (41)], negative [anhedonia/asociality: (35, 43); blunted affect: (35); alogia:
(43)] and basic symptoms (41), social and global functioning (35-37, 44), and the Strauss and Carpenter Prognostic Scale (SCPS) (38). In details, we found that the best clinical predictors recognized were schizotypal personality characteristics (35), formal thought disorders (39), specific items of the SCPS assessing quality of useful work and social relations, positive symptoms and subjective distress (38), disorganized communication (particularly, subthreshold thought disorder) both at baseline and as a trajectory of high persistent disorganized communication (40), and early adolescent social dysfunction (43), with baseline prodromal symptoms of disorganized communication, social anhedonia, suspiciousness, and diminished ideational richness that mediate the association with transition to psychosis. We found that several studies presented an increased predictive power when more variables were evaluated together. Particularly, a prediction model was developed and included positive symptoms, bizarre thinking, sleep disturbances, a schizotypal disorder, level of functioning in the past year, and years of education (44). Another study, using the median score of the global assessment of functioning scale (GAF) and the QLS scale, identified a "high" and "low" group (comprising of subjects functioning above or below median at both baseline and follow-up) and a "deterioration" group and "improving" group; Chi-square analyses showed that the low and deteriorating functioning groups were the most likely to develop FEP (45). Otherwise, Cannon et colleagues (36) found that five features contributed uniquely to the prediction of psychosis: a genetic risk for schizophrenia with recent deterioration in functioning, higher levels of unusual thought content, higher levels of suspicion/paranoia, greater social impairment, and a history of substance abuse. Predictive power was increased when prediction algorithms combining two or three of these variables were generated. Other studies have highlighted several different factors associated with transition to psychosis. A study (37) has identified five factors: year of entry into the clinic, duration of symptoms before clinic entry, baseline functioning, negative symptoms, and disorders of thought content. Another study (41) has recognized low IQ, the severity of attenuated positive symptoms, and particularly disorganized symptoms that were identified as highly predictive of functional outcome. A study (42) has identified, as the best transition predictors, selected APS (suspiciousness), negative symptoms (anhedonia/asociality), and cognitive deficits (reduced speed of information processing).

The highest PPV of $88.3 \%$ was obtained using a model that included measures of delusions, hallucinations or formal thought disorder (45). This model reached a SE of $97.3 \%$, SP of $86.5 \%$, and NPV of $96.8 \%$. The worst PPV (24\%) was produced by combining the following items of the SCPS for transition to a first psychotic episode in subjects clinically at high risk (CHR) of psychosis: most usual quality of useful work in the past year, quality of social relations, presence of thought disorder, delusions or hallucinations in the past year, and reported severity of subjective distress in past month, a predictive model that revealed an SE value of $76 \%$, SP of $57 \%$, and NPV of $93 \%$ (38).

Validation was not obtained in any clinical predictive model. 
TABLE 1 | Articles Reporting Predictive Models of Transition to Psychotic Disorder in CHR Subjects.

\begin{tabular}{|c|c|c|c|c|}
\hline Articles & $\begin{array}{c}\text { Type of CHR diagnostic instrument } \\
\text { used }\end{array}$ & $\begin{array}{c}\text { Sample of the CHR subjects (NT/ } \\
\text { T) }\end{array}$ & $\begin{array}{l}\text { Antipsychotics (patients } \\
\text { treated) }\end{array}$ & $\begin{array}{l}\text { Follow-up } \\
\text { (months) }\end{array}$ \\
\hline Mason et al. (35) & APSS, BPRS, SAPS, SANS & $37 / 37$ & No & 26 \\
\hline Cannon et al. (36) & SIPS & 209/82 & Yes & 30 \\
\hline Nelson et al. (37) & CAARMS, BPRS & $197 / 114$ & No & 60 \\
\hline Nieman et al. (38) & SIPS, BSABS-P & $207 / 37$ & Yes & 18 \\
\hline Bearden et al. (39) & SIPS & $33 / 21$ & Yes & 12 \\
\hline DeVylder et al. (40) & SIPS & $74 / 26$ & Yes & 30 \\
\hline Ziermans et al. (41) & SIPS, BSABS-P & $33 / 10$ & Yes & 72 \\
\hline $\begin{array}{l}\text { Riecher-Rössler et al. } \\
\text { (42) }\end{array}$ & BSIP, BPRS, SANS & $32 / 21$ & No & 64 \\
\hline Tarbox et al. (43) & SIPS & $192 / 78$ & $\mathrm{n} / \mathrm{a}$ & 30 \\
\hline Ruhrmann et al. (44) & SIPS, BSABS-P & $146 / 37$ & Yes & 18 \\
\hline Velthorst et al. (45) & SIPS & $119 / 28$ & No & 24 \\
\hline van Tricht et al. (46) & SIPS & $91 / 22$ & Yes & 18 \\
\hline Perkins et al. (47) & SIPS & $40 / 32$ & Yes & 24 \\
\hline Van Tricht et al. (48) & SIPS, PANSS, PAS & $43 / 18$ & $16^{\star}$ & 36 \\
\hline Ramyead et al. (49) & BSIP & $35 / 18$ & No & 36 \\
\hline Koutsouleris et al. (50) & BSIP, BPRS & $21 / 16$ & 4 & 84 \\
\hline Koutsouleris et al. (51) & BSABS & $18 / 15$ & No & 18 \\
\hline Koutsouleris et al. (52) & BPRS, SANS, PANSS & $33 / 33$ & No & 52 \\
\hline Hoffman et al. (53) & SIPS & $19 / 9$ & No & 24 \\
\hline Koutsouleris et al. (54) & CAARMS, BSABS-P & $20 / 15$ & No & 48 \\
\hline Pukrop et al. (55) & SIPS, BSABS-P & $39 / 44$ & No & 36 \\
\hline Fusar-Poli et al. (56) & CAARMS & $129 / 23$ & Yes & 24 \\
\hline Dragt et al. (57) & SIPS and BSABS-P & $53 / 19$ & Yes & 36 \\
\hline Buchy et al. (58) & SIPS & $141 / 29$ & No & 48 \\
\hline Nieman et al. (59) & SIPS, BSABS-P & $43 / 18$ & Yes & 36 \\
\hline Lencez et al. (60) & SIPS & $21 / 12$ & No & 32 \\
\hline Cornblatt et al. (61) & SIPS & $77 / 15$ & Yes & 36 \\
\hline Michel et al. (62) & SIPS, SPI-A & $53 / 44$ & Yes & 24 \\
\hline Chan et al. (63) & CAARMS & $58 / 18$ & No & 24 \\
\hline Corcoran et al. (64) & SIPS, SOPS & $42 / 7$ & $\mathrm{n} / \mathrm{a}$ & 24 \\
\hline Gschwandtner et al. (65) & BSIP, BPRS & $30 / 12$ & No & 72 \\
\hline Mittal. et al. (66) & SIPS & $66 / 24$ & 13 & 24 \\
\hline Rüsch et al. (67) & SIPS & $159 / 13$ & 33 & 12 \\
\hline Thompson et al. (68) & CAARMS & $63 / 41$ & No & 28 \\
\hline Zimmermann et al. (69) & BPRS, SANS & $15 / 13$ & 4 & 48 \\
\hline Ruhrmann et al. (44) & BSABS-P, SIPS & $208 / 37$ & 55 & 18 \\
\hline Yung et al. (70) & CASH, BPRS & $68 / 36$ & No & 12 \\
\hline Yung et al. (71) & CASH, BPRS & $29 / 20$ & No & 12 \\
\hline
\end{tabular}

APSS, the assessment of prodromal and schizotypal symptoms; BPRS, Brief Psychiatric Rating Scale; BSABS-P, The Bonn Scale for the assessment of basic symptoms- prediction list; BSIP, Basel Screening Instrument for Psychosis; CAARMS, comprehensive assessment of at risk mental states; CASH, comprehensive assessment of symptoms and history; CHR, clinical high risk; ERIraos, early recognition inventory based on the retrospective assessment of the onset of schizophrenia; HR, high risk; n/a not available; NT, nontransition; PANSS, Positive and Negative Symptoms Scale; PAS, premorbid assessment scale; PSE, present state examination; SANS; Scale for Assessment of Negative Symptoms; SAPS, Scale for Assessment of Positive Symptoms; SD, standard deviation; SIPS, structured interview for prodromal syndromes; SOPS, Scale of Prodromal Symptoms; SPI-A, Schizophrenia Proneness Instrument, Adult version; T:transition.aData are shown for the CHR subjects with a known outcome ( $n=183)$. The total group included 245 subjects.

${ }^{*} 16$ subjects treated: 9 of them nontransition and 7 transition to psychosis.

\section{Biological Predictive Models}

Seven studies have evaluated the prognostic accuracy of biological predictive models (Table 2). These studies have taken into consideration the MRI based biomarkers $(50,52)$, multivariate neuroanatomical pattern (51), electrophysiological indicators [quantitative EEG: $(46,49)$; ERP: event-related potentials: (48)], and blood analyses (47). In details, two studies took into consideration quantitative EEG $(46,49)$. Van Tricht and colleagues (46) determined quantitative EEG (QEEG) spectral power and alpha peak frequencies (APF), founding that power in theta and delta ranges and occipital-parietal APF contribute to the short-term prediction of psychosis and enable a further stratification of risk in CHR samples. Ramyead et al.
(49) assessed the individualized prediction of psychosis by detecting specific patterns of beta and gamma oscillations using machine-learning algorithms, determining that transition to psychosis could be predicted from current-source density (CSD). This study found that left superior temporal gyrus, the left inferior parietal lobule, and the precuneus most strongly contributed to the prediction of psychosis, suggesting that CSD measurements extracted from clinical resting state EEG can be useful to improve the prediction to psychosis. A study (47) took into consideration blood biomarkers, measuring expression of plasma analytes reflecting inflammation, oxidative stress, hormones, and metabolism. A "greedy algorithm" selected analytes that best distinguished individuals with clinical high- 
TABLE 2 | Prognostic Accuracy Parameters of the Predictive Models Included in the Systematic Review.

\begin{tabular}{|c|c|c|c|c|c|c|c|c|}
\hline Articles & Predictor area & $\begin{array}{c}\text { Predictive } \\
\text { model }\end{array}$ & Validation & Predictive variables (Cut-off and/or AUC) & $\begin{array}{l}\text { SE } \\
(\%)\end{array}$ & $\begin{array}{l}\text { SP } \\
(\%)\end{array}$ & $\begin{array}{l}\text { PPV } \\
(\%)\end{array}$ & $\begin{array}{r}\text { NPV } \\
(\%)\end{array}$ \\
\hline $\begin{array}{l}\text { Mason et al. } \\
\text { (35) }\end{array}$ & Clinical & $\begin{array}{l}\text { Logistic } \\
\text { regression }\end{array}$ & No & $\begin{array}{l}\text { Odd belief (SPD } \geq 1 \text { ), marked impairment in role functioning (APSS } \geq \\
\text { mild), auditory hallucinations (SAPS } \geq 2 \text { ), anhedonia/asociality (SANS } \geq 2 \text { ), } \\
\text { blunted affect (APSS } \geq \text { mild) }\end{array}$ & 84 & 86 & 86 & 84 \\
\hline \multirow[t]{4}{*}{$\begin{array}{l}\text { Cannon } \\
\text { et al. (36) }\end{array}$} & Clinical & $\begin{array}{l}\text { Cox } \\
\text { proportional } \\
\text { hazard model }\end{array}$ & No & Unusual thought content (SIPS > 3) & 56 & 62 & 48 & / \\
\hline & & & & Suspicion/paranoia (SIPS > 2) & 79 & 37 & 43 & / \\
\hline & & & & Social functioning (SIPS $<7)$ & 80 & 43 & 46 & / \\
\hline & & & & Psychosis in first-degree relatives with functional decline (GAF and SIPS) & 66 & 59 & 52 & / \\
\hline $\begin{array}{l}\text { Nelson et al. } \\
\text { (37) }\end{array}$ & Clinical & $\begin{array}{l}\text { Cox } \\
\text { proportional } \\
\text { hazard model }\end{array}$ & No & Global functioning (GAF < 44), duration symptoms (CAARMS > 738 d) & 45 & 88 & 72 & 69 \\
\hline $\begin{array}{l}\text { Nieman } \\
\text { et al. (38) }\end{array}$ & Clinical & $\begin{array}{l}\text { Cox } \\
\text { proportional } \\
\text { hazard model }\end{array}$ & No & SCPS $<49$ & 76 & 57 & 24 & 93 \\
\hline $\begin{array}{l}\text { Bearden } \\
\text { et al. (39) }\end{array}$ & Clinical & $\begin{array}{l}\text { Logistic } \\
\text { regression }\end{array}$ & No & Illogical thinking score (K-FTDS) & 69 & 71 & / & / \\
\hline \multirow[t]{3}{*}{$\begin{array}{l}\text { DeVylder } \\
\text { et al. (40) }\end{array}$} & Clinical & $\begin{array}{l}\text { Cox } \\
\text { proportional } \\
\text { hazard model }\end{array}$ & No & $\begin{array}{l}\text { Disorganized communication (SIPS }>2 \text {, AUC in the } 2 \text { through } 4 \text { range: } \\
0.64 \text { ) }\end{array}$ & 81 & 38 & 33 & 85 \\
\hline & & & & $\begin{array}{l}\text { Disorganized communication (SIPS }>3, \text { AUC in the } 2 \text { through } 4 \text { range: } \\
0.64)^{b}\end{array}$ & 62 & 62 & 36 & 82 \\
\hline & & & & $\begin{array}{l}\text { Disorganized communication score (SIPS > 4, AUC in the } 2 \text { through } 4 \\
\text { range: 0.64) }\end{array}$ & 31 & 81 & 36 & 77 \\
\hline \multirow[t]{2}{*}{$\begin{array}{l}\text { Ziermans } \\
\text { et al. (41) }\end{array}$} & Clinical & $\begin{array}{l}\text { Logistic } \\
\text { regression }\end{array}$ & No & Positive symptoms (SIPS > 11.5, AUC: 0.80) & 40 & 85 & 44 & / \\
\hline & & & & Cognitive deficits $\geq 19$ (BSABS-P $\geq 19$, AUC: 0.79) & 67 & 87 & 60 & 91 \\
\hline $\begin{array}{l}\text { Riecher- } \\
\text { Rössler } \\
\text { et al. (42) }\end{array}$ & Clinical & $\begin{array}{l}\text { Logistic } \\
\text { regression }\end{array}$ & No & Suspiciousness (BPRS:0.41, AUC: 0.72) & 70 & 72 & 61 & 79 \\
\hline \multirow[t]{2}{*}{$\begin{array}{l}\text { Tarbox et al. } \\
\text { (43) }\end{array}$} & Clinical & $\begin{array}{l}\text { Cox } \\
\text { proportional } \\
\text { hazard model }\end{array}$ & No & Alogia, anhedonia-asociality (SANS:0.33, AUC: 0.78) & 79 & 68 & / & / \\
\hline & & & & Suspiciousness (SIPS > 3) & 53 & 76 & 51 & 75 \\
\hline \multirow[t]{3}{*}{$\begin{array}{l}\text { Ruhrmann } \\
\text { et al. (44) }\end{array}$} & Clinical & $\begin{array}{l}\text { Cox } \\
\text { proportional } \\
\text { hazard model }\end{array}$ & No & Disorganized communication (SIPS > 1) & 72 & 46 & 40 & 76 \\
\hline & & & & Social anhedonia (SIPS >2) & 69 & 58 & 46 & 80 \\
\hline & & & & $\begin{array}{l}\text { Positive symptoms (SIPS }>16) \text {, bizarre thinking (SIPS > 2), schizotypal } \\
\text { personality disorder (SIPS), highest functioning score in the past year } \\
\text { (GAF-M score), sleep disturbances (SIPS }>2 \text { ), years of education, AUC: } \\
0.81\end{array}$ & 42 & 98 & 83 & 87 \\
\hline $\begin{array}{l}\text { Velthorst } \\
\text { et al. (45) }\end{array}$ & Clinical & $\begin{array}{l}\text { Logistic } \\
\text { regression }\end{array}$ & Apparent & $\begin{array}{l}\text { PANSS, with a score of } 4 \text { or more on delusions, hallucinations or formal } \\
\text { thought disorder'; having a score of } 6 \text { on any of the items of the SIPS- } \\
\text { Positive Symptoms subscales for more than } 7 \mathrm{~d} \text {. LCFA to the } 19 \text { items of } \\
\text { the SIPS. }\end{array}$ & 97.3 & 86.5 & 88.3 & 96.8 \\
\hline $\begin{array}{l}\text { Van Tricht } \\
\text { et al. (46) }\end{array}$ & Biological & $\begin{array}{l}\text { Cox } \\
\text { proportional } \\
\text { hazard model }\end{array}$ & No & $\begin{array}{l}\text { Quantitative EEG: occipital-parietal individual alpha peak frequency, frontal } \\
\text { delta and theta power. }\end{array}$ & 46 & 87 & 56 & 87 \\
\hline $\begin{array}{l}\text { Perkins et al. E } \\
(47)\end{array}$ & Biological & $\begin{array}{l}\text { Greedy } \\
\text { algorithm }\end{array}$ & Internal & $\begin{array}{l}\text { Blood biomarker: interleukin-1B, GH, KIT ligand, interleukin-8, matrix } \\
\text { metalloproteinase-7, interleukin-7, resistin, chemokine [C-c motif] ligand8, } \\
\text { immunoglobulin E, coagulation factor VII, TSH, malondialdehyde-modified } \\
\text { low-density lipoprotein, apolipoproteinD, uromodulin and cortisol (AUC: } \\
\text { 0.88) }\end{array}$ & 60 & 90 & 72 & 84 \\
\hline $\begin{array}{l}\text { Van Tricht } \\
\text { et al. (48) }\end{array}$ & Biological & $\begin{array}{l}\text { Cox } \\
\text { proportional } \\
\text { hazard model }\end{array}$ & No & ERP: P300 (Amplitude < 14.7 microvolt) & 83 & 79 & / & / \\
\hline $\begin{array}{l}\text { Ramyead et al. } \\
\text { (49) }\end{array}$ & Biological & LASSO & Internal & 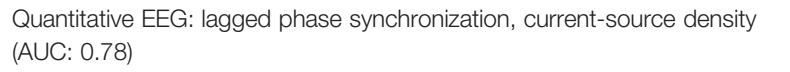 & 58 & 83 & / & / \\
\hline $\begin{array}{l}\text { Koutsouleris } \\
\text { et al. (50) }\end{array}$ & Biological & $\begin{array}{l}\text { Binary SVM } \\
\text { with radial }\end{array}$ & $\begin{array}{l}\text { Internal with } \\
\text { nested } \\
\text { repeated 10- }\end{array}$ & $\begin{array}{l}\text { MRI-based biomarkers (The neuroanatomical decision functions } \\
\text { underlying these results particularly involved the prefrontal perisylvian and } \\
\text { subcortical brain structures) }\end{array}$ & 81.0 & 87.5 & 77.8 & 89.5 \\
\hline
\end{tabular}


TABLE 2 | Continued

\begin{tabular}{|c|c|c|c|c|c|c|c|c|}
\hline Articles & Predictor area & $\begin{array}{l}\text { Predictive } \\
\text { model }\end{array}$ & Validation & Predictive variables (Cut-off and/or AUC) & $\begin{array}{l}\text { SE } \\
(\%)\end{array}$ & $\begin{array}{l}\text { SP } \\
(\%)\end{array}$ & $\begin{array}{l}\text { PPV } \\
(\%)\end{array}$ & $\begin{array}{l}\text { NPV } \\
(\%)\end{array}$ \\
\hline & & $\begin{array}{l}\text { basis } \\
\text { function }\end{array}$ & $\begin{array}{l}\text { fold cross- } \\
\text { validation }\end{array}$ & & & & & \\
\hline $\begin{array}{l}\text { Koutsouleris } \\
\text { et al. (51) }\end{array}$ & Biological & $\begin{array}{l}\text { Binary SVM } \\
\text { with radial } \\
\text { basis } \\
\text { function }\end{array}$ & $\begin{array}{l}\text { Internal with } \\
5 \text {-fold cross- } \\
\text { validation }\end{array}$ & $\begin{array}{l}\text { Multivariate neuroanatomical pattern classification performed on the } \\
\text { structural magnetic resonance imaging data }\end{array}$ & 83 & 80 & 83 & 80 \\
\hline $\begin{array}{l}\text { Koutsouleris } \\
\text { et al. (52) }\end{array}$ & Biological & SVM & Internal & $\begin{array}{l}\text { Gray matter volume reduction (dorsomedial, ventromedial, and } \\
\text { orbitofrontal areas extending to the cingulate and right intra- and } \\
\text { perisylvian structures }\end{array}$ & 76 & 85 & 83 & 78 \\
\hline $\begin{array}{l}\text { Hoffman et al. } \\
\text { (53) }\end{array}$ & Neurocognitive & $\begin{array}{l}\text { Cox } \\
\text { proportional } \\
\text { hazard model }\end{array}$ & No & Length of speech illusion (babble task $\geq 4$ ) & 89 & 90 & 80 & 94 \\
\hline $\begin{array}{l}\text { Koutsouleris } \\
\text { et al. (54) }\end{array}$ & Neurocognitive & SVM & Internal & $\begin{array}{l}\text { Verbal and executive functioning (MWT-B, DST, TMT-B, RAVLT-DR, and } \\
\text { RAVLT-Ret) }\end{array}$ & 75 & 80 & 83 & 71 \\
\hline $\begin{array}{l}\text { Riecher- } \\
\text { Rössler et al. } \\
\text { (43) }\end{array}$ & Neurocognitive & $\begin{array}{l}\text { Logistic } \\
\text { regression }\end{array}$ & No & $\begin{array}{l}\text { Verbal IQ and attention (MWT/TAP Go/NoGo false alarm: 0.38, AUC: } \\
0.62 \text { ) }\end{array}$ & 80 & 59 & 57 & 83 \\
\hline $\begin{array}{l}\text { Pukrop et al. } \\
\text { (55) }\end{array}$ & Neurocognitive & $\begin{array}{l}\text { Logistic } \\
\text { regression }\end{array}$ & No & $\begin{array}{l}\text { Verbal memory-delayed recall (Auditory Verbal Learning Test), verbal IQ } \\
\text { (Multiple Choice Vocabulary Test), verbal memory-immediate recall } \\
\text { (Auditory Verbal Learning Test) and processing speed (DST) }\end{array}$ & 75 & 79 & 80 & 74 \\
\hline $\begin{array}{l}\text { Ziermans et al. } \\
(41)\end{array}$ & Neurocognitive & $\begin{array}{l}\text { Logistic } \\
\text { regression }\end{array}$ & No & IQ (Wechsler Intelligence Scales < 86.5, AUC: 0.77) & 40 & 97 & 80 & 84 \\
\hline $\begin{array}{l}\text { Fusar-Poli } \\
\text { et al. (56) }\end{array}$ & Environmental & Log-rank test & No & Unemployment ("yes/no" assessed with unstandardized questionnaire) & 57 & 61 & 20 & 89 \\
\hline \multirow[t]{2}{*}{$\begin{array}{l}\text { Dragt et al. } \\
(57)\end{array}$} & Environmental & $\begin{array}{l}\text { Cox } \\
\text { proportional } \\
\text { hazard model }\end{array}$ & No & Urbanicity (BDF, $\leq 100000$ inhabitants), impaired & 63 & 88 & 63 & 88 \\
\hline & & & & $\begin{array}{l}\text { social-sexual aspects, age 12-15 (PAS), impaired } \\
\text { social-personal adjustment, general (PAS) }\end{array}$ & & & & \\
\hline $\begin{array}{l}\text { Tarbox et al. } \\
(43)\end{array}$ & Environmental & $\begin{array}{l}\text { Cox } \\
\text { proportional } \\
\text { hazard }\end{array}$ & No & Early adolescent social maladjustment (PAS > 2) & 50 & 71 & 46 & 72 \\
\hline $\begin{array}{l}\text { Buchy et al. } \\
(58)\end{array}$ & Environmental & $\begin{array}{l}\text { Cox } \\
\text { proportional } \\
\text { hazard }\end{array}$ & No & Alcohol use ("yes/no" AUS/DUS) & 69 & 81 & 26 & 90 \\
\hline $\begin{array}{l}\text { Cannon et al. } \\
(36)\end{array}$ & Environmental & $\begin{array}{l}\text { Cox } \\
\text { proportional } \\
\text { hazard model }\end{array}$ & No & $\begin{array}{l}\text { Abuse of alcohol, hypnotics, cannabis, amphetamines, opiates, cocaine, } \\
\text { hallucinogens ("yes/no" as assessed by the Structured Clinical Interview } \\
\text { for DSM-IV or the Schedule for Affective Disorders and Schizophrenia for } \\
\text { School-Age Children) }\end{array}$ & 29 & 83 & 43 & / \\
\hline $\begin{array}{l}\text { Ziermans et al. } \\
(41)\end{array}$ & Combination & $\begin{array}{l}\text { Logistic } \\
\text { regression }\end{array}$ & No & $\begin{array}{l}\text { Positive symptoms (SIPS > 11.5) and IQ (Wechsler Intelligence Scales } \leq \\
\text { 86.5) (AUC: 0.82) }\end{array}$ & 50 & 91 & 63 & 86 \\
\hline $\begin{array}{l}\text { Riecher- } \\
\text { Rössler et al. } \\
\text { (42) }\end{array}$ & Combination & $\begin{array}{l}\text { Logistic } \\
\text { regression }\end{array}$ & Internal & $\begin{array}{l}\text { Suspiciousness (BPRS), anhedonia-asociality (SANS) and attention (TAP } \\
\text { Go/NoGo false alarm) (cut-off: } 0.41, \text { AUC: } 0.87 \text { ) }\end{array}$ & 83 & 79 & 71 & 86 \\
\hline $\begin{array}{l}\text { Nieman et al. } \\
\text { (59) }\end{array}$ & Combination & $\begin{array}{l}\text { Cox } \\
\text { proportional } \\
\text { hazard } \\
\text { model }\end{array}$ & Internal & P300 amplitude (ERP), social-personal adjustment & 78 & 88 & 74 & 90 \\
\hline $\begin{array}{l}\text { Lencz et al. } \\
(60)\end{array}$ & Combination & $\begin{array}{l}\text { Logistic } \\
\text { regression }\end{array}$ & No & $\begin{array}{l}\text { Verbal memory (Wechsler Memory Scale) and positive symptoms (SIPS) } \\
\text { (AUC: 0.43) }\end{array}$ & 82 & 79 & 69 & 88 \\
\hline \multirow[t]{4}{*}{$\begin{array}{l}\text { Tarbox et al. } \\
\text { (43) }\end{array}$} & Combination & $\begin{array}{l}\text { Cox } \\
\text { proportional } \\
\text { hazard model }\end{array}$ & No & $\begin{array}{l}\text { Early adolescent social maladjustment (PAS > 2), suspiciousness (SIPS > } \\
\text { 3) }\end{array}$ & 28 & 92 & 59 & 70 \\
\hline & & & & $\begin{array}{l}\text { Early adolescent social maladjustment (PAS > 2), disorganized } \\
\text { communication (SIPS > 1) }\end{array}$ & 42 & 82 & 51 & 72 \\
\hline & & & & $\begin{array}{l}\text { Early adolescent social maladjustment (PAS }>2 \text { ), social anhedonia (SIPS } \\
>2 \text { ) }\end{array}$ & 43 & 78 & 49 & 72 \\
\hline & & & & $\begin{array}{l}\text { Early adolescent social maladjustment (PAS > 2), ideational richness } \\
(\text { SIPS }>0)\end{array}$ & 32 & 85 & 50 & 70 \\
\hline $\begin{array}{l}\text { Cornblatt et al. } \\
(61)\end{array}$ & Combination & $\begin{array}{l}\text { Cox } \\
\text { proportional } \\
\text { hazard model }\end{array}$ & No & $\begin{array}{l}\text { Disorganized communication (SIPS }>2 \text { ), suspiciousness }(\mathrm{SIPS}=5) \text {, } \\
\text { verbal memory deficit } 2 \text { SD below normal, declining social functioning } \\
\text { (Global Functioning: Social scale) (AUC: 0.92) }\end{array}$ & 60 & 97 & 82 & 93 \\
\hline
\end{tabular}


TABLE 2 | Continued

\begin{tabular}{|c|c|c|c|c|c|c|c|c|}
\hline Articles & Predictor area & $\begin{array}{l}\text { Predictive } \\
\text { model }\end{array}$ & Validation & Predictive variables (Cut-off and/or AUC) & $\begin{array}{l}\text { SE } \\
(\%)\end{array}$ & $\begin{array}{l}\text { SP } \\
(\%)\end{array}$ & $\begin{array}{l}\text { PPV } \\
(\%)\end{array}$ & $\begin{array}{l}\text { NPV } \\
(\%)\end{array}$ \\
\hline $\begin{array}{l}\text { Cannon et al. } \\
\text { (36) }\end{array}$ & Combination & $\begin{array}{l}\text { Cox } \\
\text { proportional } \\
\text { hazard model }\end{array}$ & No & $\begin{array}{l}\text { Psychosis in first-degree relatives with functional decline (SIPS and GAF), } \\
\text { unusual thought content (SIPS }>3 \text { ), social functioning (SIPS }<7 \text { ) }\end{array}$ & 30 & 90 & 81 & / \\
\hline $\begin{array}{l}\text { Michel et al. } \\
\text { (62) }\end{array}$ & Combination & $\begin{array}{l}\text { Cox } \\
\text { proportional } \\
\text { hazard }\end{array}$ & Internal & UHR criteria (SIPS), DST deficit t-score < 40, COGDIS criteria (BSABS-P) & 57 & 66 & 58 & 65 \\
\hline Chan et al. (63) & Combination & LASSO & $\begin{array}{l}10 \text {-fold cross } \\
\text { validation }\end{array}$ & 22-Analyte panel, CAARMS-positive subscale (AUC:0.90) & 89 & 79 & 57 & 96 \\
\hline $\begin{array}{l}\text { Corcoran et al. } \\
(64)\end{array}$ & Combination & $\begin{array}{l}\text { Logistic } \\
\text { regression }\end{array}$ & Apparent & $\begin{array}{l}\text { Facial emotion discrimination (EMODIFF), Facial emotion recognition } \\
\text { (ER40), Negative symptoms (AUC:0.99) }\end{array}$ & 86 & 98 & 86 & 98 \\
\hline $\begin{array}{l}\text { Gschwandtner } \\
\text { et al. (65) }\end{array}$ & Combination & $\begin{array}{l}\text { Logistic } \\
\text { regression } \\
\text { model }\end{array}$ & No & EEG and general psychopathology (SANS and BPRS) (AUC=0.81) & 82 & 73 & / & / \\
\hline Mittal et al. (66) & ) Combination & $\begin{array}{l}\text { Linear } \\
\text { discriminant } \\
\text { analysis }\end{array}$ & $\begin{array}{l}\text { Internal with } \\
\text { leave one } \\
\text { out cross- } \\
\text { validation }\end{array}$ & $\begin{array}{l}\text { Movement abnormalities (Dyskinesia Identification System: Condensed } \\
\text { User), functional domains (WAIS-III, WISC-III), Neurocognition (FSIQ, } \\
\text { vocabulary, matrix reasoning, block design, Logical memory I, Logical } \\
\text { Memory II }\end{array}$ & 76.0 & 60 & 86.3 & 43 \\
\hline $\begin{array}{l}\text { Rusch et al. } \\
\text { (67) }\end{array}$ & Combination & $\begin{array}{l}\text { Logistic } \\
\text { regression } \\
\text { and cox } \\
\text { proportional } \\
\text { hazard model }\end{array}$ & Apparent & $\begin{array}{l}\text { Positive and Negative symptoms (PANSS), perceived stigma-related harm } \\
\text { (validated 8-item self-report measure based on Lazarus and Folkman's } \\
\text { (1984) conceptualization of stress appraisal processes; using the median } \\
\text { of as a cut off) }\end{array}$ & 58 & 98 & / & / \\
\hline $\begin{array}{l}\text { Thompson } \\
\text { et al. (68) }\end{array}$ & Combination & $\begin{array}{l}\text { Cox } \\
\text { proportional } \\
\text { hazard model }\end{array}$ & Apparent & $\begin{array}{l}\text { Genetic risk with functional decline; high unusual thought content score } \\
\text { ( }>3 \text { on the SIPS); high suspicion/paranoia score ( }>2 \text { on the SIPS); low } \\
\text { social functioning ( }<7 \text { on the Social Functioning Scale) and history of } \\
\text { substance abuse. }\end{array}$ & 37.3 & 87.2 & 65.4 & 68.2 \\
\hline $\begin{array}{l}\text { Zimmermann } \\
\text { et al. (69) }\end{array}$ & Combination & $\begin{array}{l}\text { Logistic } \\
\text { regression }\end{array}$ & Apparent & $\begin{array}{l}\text { Negative symptom scale (SANS) and EEG spectral data (EEG power in } \\
\text { seven bands: delta, theta, alpha1, alpha2, beta1, beta2, beta3) }\end{array}$ & 92 & 87 & 86 & 93 \\
\hline $\begin{array}{l}\text { Ruhrmann } \\
\text { et al. (44) }\end{array}$ & Combination & $\begin{array}{l}\text { Cox } \\
\text { proportion } \\
\text { hazard model }\end{array}$ & Apparent & $\begin{array}{l}\text { SIPS-Positive score, bizarre thinking, sleep disturbances, schizotypal } \\
\text { personality disorder (according to SIPS) highest GAF-M score in the past } \\
\text { year, and years of education (AUC: } 80.8 \text { ) }\end{array}$ & 41.7 & 97.9 & 83.3 & 87.0 \\
\hline Yung et al. (70) & Combination & $\begin{array}{l}\text { Cox } \\
\text { proportional } \\
\text { hazard model }\end{array}$ & Apparent & $\begin{array}{l}\text { Belonging to both the Trait and Attenuated Groups, Duration }>5 \text { years, } \\
\text { SANS attention }>2, G A F<40\end{array}$ & 60.0 & 92.6 & 80.8 & 81.8 \\
\hline Yung et al. (71) & Combination & $\begin{array}{l}\text { Cox } \\
\text { proportional } \\
\text { hazard } \\
\text { model }\end{array}$ & Apparent & $\begin{array}{l}\text { Duration of symptoms }>900 \mathrm{~d}, \text { GAF } \\
\text { score }<51 \text {, BPRS total }>15 \text {, BPRS } \\
\text { psychotic subscale }>2 \text {, SANS attention } \\
\text { score }>1 \text { and HRSD }>18\end{array}$ & 86 & 91 & 80 & 94 \\
\hline
\end{tabular}

CAARMS, Comprehensive Assessment of At-Risk Mental State; LCFA, Latent Class Factor Analysis; NPV, negative predictive value; PPV, positive predictive value; SE, sensibility; SP, specificity.

Adapted by Schmidt et al. (28).

APSS, the assessment of prodromal and schizotypal symptoms; AUC, area under the curve; AUS/DUS, The Alcohol and Drug Use Scale; BDF, basic data form; BPRS,

Brief Psychiatric Rating Scale; BSABS-P, The Bonn Scale for the assessment of basic symptoms-prediction list; CAARMS, comprehensive assessment of at risk mental states; CODGIS, cognitive disturbances; DST, digit symbol test; EEG, electroencephalogram; ERP, event-related potentials; GAF: global assessment of functioning; HRSD, Hamilton Rating; Scale for Depression; K-FTDS, Kiddie-Formal Thought Disorder Scale; LASSO, least absolute shrinkage and selection operator; MWT, Mehrfachwahl- Wortschatz test; NPV, negative predictive value; PAS, Premorbid Adjustment Scale; PPV, predictive positive value; RAVLT-DR, Rey Auditory Verbal Learning-delayed recall;

RAVLT-Ret, Rey Auditory Verbal Learning-retention; SANS, Scale for Assessment of Negative Symptoms; SCPS, Strauss and Carpenter Prognostic Scale, score; SD, standard deviation; SE, sensitivity; SFS, social functioning scale; SP, specificity; SPD, Schizotypal Personality Disorder subscale of the International Personality Disorder Examination; SIPS, structured interview for prodromal syndromes; SVM, support vector machine; TAP, Testbatterie zur Aufmerksamkeitspïfung; TMT, trail-making test; WISC-III, Wechsler Intelligence Scales for Children 3rd ed. for participants ages 11 to 15; WAIS-III, Wechsler Adult Intelligence Scales, 3rd ed; FSIQ. Full Scale Intelligence Quotient; HRSD, Hamilton Rating Scale for Depression.

${ }^{a}$ Cut-off scores for determining sensitivity, specificity, and accuracy values were derived from the receiver operating characteristic curve.

${ }^{b}$ The Youden Index (maximal value for sensitivity + specificity - 1) was 0.24 with the optimal cut point of a score of 3 for baseline disorganized communication.

${ }^{c}$ This model included 58 (of 61) CHR subjects.

risk symptoms who developed psychosis. The classifier included 15 analytes (selected from 117). These results support the hypothesis that inflammation, oxidative stress, and dysregulation of hypothalamic-pituitary axes may be prominent in the earliest stages of psychosis and could lead to develop a multiplex blood assay with a potential for high clinical utility. A study (48) analyzed abnormalities on neuroimaging and neuropsychological examinations before the onset of a first psychotic episode, founding that reduced P3 amplitudes (a scalprecorded late ERP, occurring approximately $300 \mathrm{~ms}$ after an attended unusual or task-relevant stimulus.) were identified as the best predictor for subsequent psychosis in the UHR group. The $\mathrm{P} 3$ reduction was related to increased social anhedonia and withdrawal and a lower global assessment of social functioning and social personal adjustment. Different studies (50, 52) concentrated their efforts in individuate MRI biomarkers: the 
first study (50) found that the neuroanatomical decision functions underlying these results particularly involved the prefrontal perisylvian and subcortical brain structures and the second (52) found that the predictor's decision function involved grey matter volume alterations in prefrontal, perisylvian, and subcortical structures, supporting the idea of the existence of a cross-center neuroanatomical signature of emerging psychosis enabling individualized risk staging across different high-risk populations. Finally, another study (51) developed a multivariate neuroanatomical pattern classification on the structural magnetic resonance imaging data of individuals, in order to help predicting transition to psychosis.

The highest PPV of $83 \%$ was reached using the predictive variable of the grey matter volumes (grey matter volume alterations in prefrontal, perisylvian, and subcortical structures), with a SE of $76 \%$, SP of $85 \%$, and NPV of $78 \%$ (52). This review has been internally validated. However, the study sample was 66 subjects, constituting a rather small sample. Globally, five of these studies $(47,49-52)$ were cross validated and two were not $(46,48)$.

The worst PPV (77.8\%) resulted from a predictive model including MRI-based biomarkers. This predictive model yielded an SE of $81 \%$, SP of $87.5 \%$, and NPV of $89.5 \%$ (50).

\section{Neurocognitive Predictive Models}

Five studies have analyzed the prognostic accuracy of cognitive predictive models (Table 2 ). These studies have provided measurements of IQ $(41,42,55)$, verbal memory $(54,55)$, attention (42), speech perception (53), executive functioning (54), and processing speed $(54,55)$.

One of the studies (41) showed that low IQ was the single neurocognitive parameter that discriminated patients at ultra-high risk converted to psychosis from individuals who did not. The severity of attenuated positive symptoms was the only significant predictor of a transition to psychosis and disorganized symptoms were highly predictive of functional outcome.

Another study (42) showed that best transition predictors were selected APS (suspiciousness), negative symptoms (anhedonia/asociality), and cognitive deficits (reduced speed of information processing). Prediction of transitions could be enhanced by a stronger weighting of certain early symptoms and by inserting neurocognitive tests into a stepwise risk assessment. Therefore, this study uses neurocognition in addition to clinical parameters for predicting transition to psychosis. Hoffman and colleagues (53) highlighted that elevated LSI (length of speech illusion) scores indicated increased risk of transition to psychotic disorders when individual participating to the study were not taking olanzapine. A further study (54) has demonstrated that patients at risk of transition to psychosis could be identified on an individual basis by evaluating neurocognitive test batteries using multivariate pattern recognition. In another study (55) several cognitive domains were identified as indicators of vulnerability to psychosis. In addition, the results of the article suggest that subtle deficits in verbal abilities (working and long- term memory, executive and intellectual functions) and decreased speed of processing may help to predict transition to psychosis.

Considering verbal and executive functioning in the predictive model (neuropsychological functions were assessed with a cross-domain neuropsychological test battery comprising nine standardized tests that evaluated premorbid verbal IQ, processing speed, working memory, verbal learning and memory, executive functions, and verbal fluency), the highest PPV of $83 \%$ could be obtained with a value of SE equal to $75 \%$, SP equal to $80 \%$, and NPV equal to $71 \%$ (54). This model is the only one that has been validated in this domain, with an internal validation. However, the sample of the study is quite small, resulting in 35 subjects. The worst PPV of 57\% was achieved by using a model including verbal IQ and attention (42). This model yielded an SE of $80 \%$, SP of $59 \%$, and NPV of $83 \%$ (42).

\section{Environmental Predictive Models}

The prognostic accuracy of environmental predictive models was evaluated in five papers (Table 2). These models have taken into consideration substance abuse $(36,58)$, unemployment (56), urbanity (57), social-sexual aspects (57), and social maladjustments $(43,57)$.

Two studies analyzed substance abuse $(36,58)$. Buchy et al. (58) and demonstrated that low use of alcohol contributed to the prediction of psychosis. This study has also highlighted that prediction algorithms including associations of additional baseline variables known to be associated with psychotic transition increase predictive power compared with substance use alone. Cannon et al. (36) found that different features contributed to the prediction of psychosis, including clinical features and a history of substance abuse (alcohol, hypnotics, cannabis, amphetamines, opiates, cocaine, hallucinogens): predictive power was enhanced when prediction algorithms combining two or three of these variables were developed. Tarbox et al. (43) identified that early adolescent social maladjustment and baseline suspiciousness together demonstrated moderate positive predictive power (59\%) and high specificity (92.1\%) in predicting transition to psychosis. A study (57) has identified urbanicity, social-sexual aspects, and social-personal adjustment as predictors of transition to psychosis.

Another study (56) showed that unemployment at the first contact with the prodromal service may be a risk factor for the development of a psychotic episode.

The best predictive model was obtained in a study conducted on 72 subjects, with values of PPV, SE, SP, and NPV of $63 \%, 63 \%, 88 \%$, and $88 \%$, respectively (57). Measures of urbanity, social-sexual aspects, and social and personal adjustment were significant predictors $(\mathrm{P}<.001)$. The worst PPV of $26 \%$ was achieved by using a model evaluating alcohol use ("yes/no"). This model yielded an SE of $69 \%$, SP of $81 \%$, and NPV of $90 \%$ (58).

There are no predicting models evaluating environmental factors that have been validated. 


\section{Combinations of Predictive Models}

Eighteen studies (36, 41-44, 59-71) have evaluated prognostic accuracy combining different predictive models across domains (Table 2).

Some of these studies concentrated their efforts in develop predictive models combining symptomatology and neurophysiology $(59,65,69)$. The first study (59) combined different predictive models, suggesting that predicting transition to psychosis could be improved with a model including premorbid adjustment and information-processing variables (specifically parietal P300 amplitude) in a multistep algorithm combining risk detection and stratification. A second study (65) demonstrated that patients who develop psychosis showed significantly more pathological EEG abnormalities than subjects who did not, located more frequently in temporal or frontotemporal regions of the brain. The specificity of the prediction of psychosis could be increased from 59 to $73 \%$ by considering EEG pathology in addition to psychopathology alone. Zimmermann and colleagues (69) have shown that SANS score in combination with EEG power in four bands (delta, theta, beta1, and beta2 bands), respectively, predicted transition significantly in 13 individuals with later transition to psychosis.

Other research studied predictive models focusing on symptomatology and functioning (44, 61, 68, 70, 71). A prediction model was developed including positive symptoms, bizarre thinking, sleep disturbances, a schizotypal disorder, level of functioning in the past year, and years of education (44). Another study (61) developed a final predictor model, with a positive predictive validity of $81.8 \%$, consisted of four variables: disorganized communication, suspiciousness, verbal memory deficits, and decline in social functioning during follow-up. A study (68) found three variables associated with transition to psychosis: high unusual thought content scores; low functioning; and having genetic risk with functional decline. Using a combination of two out of three of these features, the predictive validity of determining whether an individual develops psychosis was improved, although using this method the probability of a person not developing psychotic disorder is still quite high at $35 \%$. A study (70) yielded a method of psychosis prediction at 12 months, identifying the following as predictors: poor functioning, long duration of symptoms, high levels of depression, and reduced attention. A combination of family history of psychosis, a recent significant decrease in functioning and recent experience of subthreshold psychotic symptoms was also predictive of psychosis. A study (71) developed a strategy for predicting transition to psychosis, within a relatively brief follow-up period (12 months), combining some highly significant predictors of psychosis: long duration of prodromal symptoms, poor functioning at intake, low-grade psychotic symptoms, depression, and disorganization. A study (67) has developed a predictive model focusing on individuals functioning and stigma. Specifically, this study (67) showed that more perceived stigma stress at baseline predicted transition to schizophrenia after adjusting for age, gender, symptoms (positive and negative symptoms), and functioning. Other studies concentrate on cognitive deficits and symptomatology $(41,42,60,62,66)$. Another study (41) has identified low IQ, the severity of attenuated positive symptoms, and particularly disorganized symptoms as highly predictive of functional outcome. A study (42) has identified as the best transition predictors, selected APS (suspiciousness), negative symptoms (anhedonia/asociality), and cognitive deficits (reduced speed of information processing). A study (60) demonstrated that prodromal patients (with APS) who later developed psychosis had significantly lower verbal memory scores at baseline, suggesting that verbal memory deficits can represent an important risk marker of transition to psychosis, possibly indicating the presence of a prefrontalhippocampal neurodevelopmental abnormality. A study (62) found that the combination of a processing speed deficit (digit symbol test) and at-risk criteria (APS plus subjective cognitive disturbances) provides an optimized stratified risk assessment to develop psychosis. A research (66) has studied movement abnormalities and cognitive deficits demonstrating that elevated dyskinetic movements in the upper-body region were correlated with deficits in domains of verbal comprehension, perceptual organization, and both immediate and delayed auditory memory. Further, discriminant function analyses indicated that baseline movement abnormalities and neurocognitive deficits significantly classified subjects at risk to develop psychosis $(72.3 \%)$. Results support a common corticostriato-pallido-thalamic circuit irregularity, underlying both movement abnormalities and cognitive deficits in individuals at high risk for psychosis.

Another study focused on maladjustment of individuals at high-risk to develop psychosis. Tarbox et al. (43) identified that early adolescent social maladjustment and baseline suspiciousness together demonstrated moderate positive predictive power (59\%) and high specificity $(92.1 \%)$ in predicting transition to psychosis. It uses also as predictor of transition to psychosis the early adolescent social dysfunction. Other research was carried out in the field of substance abuse. Particularly, Cannon et al. (36) found that different features contributed to the prediction of psychosis, including clinical features and a history of substance abuse (alcohol, hypnotics, cannabis, amphetamines, opiates, cocaine, hallucinogens): predictive power was enhanced when prediction algorithms combining two or three of these variables were developed. Another interesting field that has been faced was about predictive models combining biology and symptomatology. In details, a study (63) developed a combined molecular/symptombased test. The authors described the development of a serum biomarker test for the identification of individuals at risk of transition to psychosis based on multiplex immunoassay profiling analysis of 957 serum samples, identifying and validating an optimal panel of 26 biomarkers that best discriminated patients and controls. The performance increased further incorporating the CAARMS (Comprehensive Assessment of At-Risk Mental State) positive subscale symptom scores into the model. Finally, attention was laid on emotion recognition. Specifically, a study (64) showed how deficits in 
emotion recognition significantly identify subjects who develop psychosis. The authors, moreover, demonstrate that the best classification model for schizophrenia onset included both face emotion processing (facial emotion discrimination and recognition) and negative symptoms. The highest PPV (86.3\%) was obtained in a study (66) that took into consideration movement abnormalities, functional domains and neurocognition, with values of SE of $76 \%$, SP of $60 \%$, and NPV of $43 \%$.

The worst PPV of $49 \%$ was achieved using early adolescent social maladjustment and baseline suspiciousness together as the predictive variables, which produced an SE of $43 \%$, SP of $78 \%$, and NPV of $72 \%$ (43).

\section{DISCUSSION}

Although the field of risk prediction in mental health lags behind other areas of medicine, some promising studies have been conducted to begin to ascertain the operative combinations of risk factors for a number of psychiatric disorders (72). These models must be successfully replicated and validated in multiple samples, external to the one used for the model development phase. This often takes many years to be achieved. The use of risk prediction models must be thoroughly evidence based, with research demonstrating that the model is reliable and applicable to the intended populations of individuals (73).

The prediction and prevention of psychotic disorders should include a two-step approach: one step aimed at the identification of individuals in CHR phase, the other aimed to further stratify risk so that "indicated preventive interventions" can be given to patients in the highest risk stratum in an even more targeted and intensive way.

The present review wants to extend the results of a recent review of Schmidt et al. (28). Our review evaluated a total of 38 studies, encompassing clinical, biological, neurocognitive, environmental, or combinations of predictive models from various domains.

Four main findings should be highlighted.

First, while the highest PPVs in clinical (35), biological (51, 52), neurocognitive (54), and combined (66) predictive models were quite high (all above 83), the highest PPV in environmental predictive models was relatively low (63\%) (57). This data could be due to the heterogeneity in the environmental factors included in the studies. Moreover, the examined environmental factors were mostly those that have been related with psychotic disorders, particularly substance abuse, urbanicity, and social maladjustment, so that it is possible that their specificity in detecting transition risk to psychosis of $\mathrm{CHR}$ is relatively poor, as outlined by a recent meta-analysis (21).

Moreover, regarding the neurocognition, while many previous studies have suggested that it is an important factor in predicting transition to psychosis, there is significant heterogeneity regarding the specific domains implicated: measurements of IQ, verbal memory, attention, speech perception, executive functioning, and processing speed $(74,75)$.

Second, none of the combined models showed a superiority when compared with more parsimonious models (using only neurocognitive, clinical, biological, or environmental factors).
Thus, based on this data, it could be inferred that a strong PPV can be reached making use of psychopathological or neurocognitive data alone, therefore this approach should be preferred to, i.e., extensive neuroimaging batteries. However, a study (28) estimating the theoretical PPV of a sequential threestage testing (that contained various combinations of three models predicting transition to psychosis, eg, electroencephalography/ clinical, images taken from MRI, and blood indicators) following the initial CHR assessment, has shown that the highest value of PPV was obtained when using in sequence a combined model (clinical + EEG) and two biological models (structural MRI and blood indicators). Particularly, PPV reached a value of $98 \%$ for subjects with three positive tests, $71-82 \%$ for subjects with two positive complementary tests, $12 \%-21 \%$ for subjects with one positive complementary test, and $1 \%$ for subjects without any positive tests. This study could indicate that testing in sequence CHR individuals with models of prediction psychosis onset across multiple domains could substantially enhance psychosis prediction after the initial CHR assessment. Thus, multistage sequential testing enables individual risk stratification of $\mathrm{CHR}$ subjects to be made and improve prediction of transition to psychosis. Third, it should be highlighted that only a few studies have tried to replicate directly each other's risk algorithms. Consequently, most published predictive performance estimates are likely to be considerably overoptimistic. Only ten studies have used a strict prognostic accuracy method matching appropriate predictive models provided of internal validation. Some of the studies presented an "apparent validation", obtained on sample used to develop model, leading to strongly overoptimistic results. The majority part of the studies lacked sufficient details to precisely apply the model in a new dataset and this can be partly explained by the fact that there are no models externally validated.

In order to create rigorous risk prediction models, validation is one of the most important elements. A useful prediction model should give accurate estimates of risk, that can be used from the physicians to help them in clinical management and decision making. Moreover, this model should have a core role in predict individuals' outcome and cost-effectiveness of care. There is a substantial difference between models with internal and external validation. When new individuals were subjected to predictive model provided of internally validation, the performance is was lower than the one observed in the sample used to develop the model (76).

Fourth, our review found that poor conduct and reporting were quite common in both predictor finding and model developed studies. The results of our review highlight that one of the biggest limitations is that most of the studies were based on small sample sizes and number of events (particularly patients with transitions to psychosis) relative to the number of evaluated predictor variables. Small number of evaluated predictor variables ratios enhance the risk of overestimating the performance of the model, if it is developed and assessed in the same sample. When sample sizes are small, as is it frequently occurs in the field of prediction of psychosis research, their performance advantage resulting from the increased ability to capture the true underlying relationship 
between predictors and response might not be high enough to compensate for the increased risk to overestimate.

Prediction in psychiatry needs to be considered a core aspect for testing hypotheses regarding clinically relevant issues (77). However, there are different problems that have to be faced before develop risk prediction models in psychiatry: one of them is the lack of availability of biological markers of illness; another is the idea that a particular discrimination value (e.g., an AUC threshold of 0.80 ) is required before clinical adoption. Indeed, in most prediction algorithms, including those regarding the Framingham risk score (FRS), the AUC often ranges from 0.75 to 0.80 (78).

Nevertheless, it's clear that a risk prediction model is useful only if early and preventive intervention are available and effective to prevent individual at high risk in developing disease. The use of validated risk prediction algorithms, despite being available, has delayed in primary care (79). If effective predictive models were designed, all the efforts should be done to make them useful and suitable for clinicians. In fact, quantification of validated prediction model impact in clinical care should be the target to be reached for implementation of these models. Though, impact studies are even less frequently performed than validation studies, as it can be elicited from literature (80).

The research about risk prediction models should progress together with the development of preventive interventions, i.e., long-chain $\omega$ - 3 polyunsaturated fatty acids (PUFAs). Although $\omega$-3 PUFAs treatment is attractive for prevention from a pathophysiologic perspective, preventive efficacy of $\omega-3$ PUFAs for psychosis had been demonstrated in one singlesite randomized, double-blind, placebo controlled trial which has compared $\omega-3$ PUFAs with placebo (81), and later confirmed in a naturalistic, long-term follow-up (82). However, two large replication trials, the NEURAPRO trial (83) and the NAPLS-2 trial (84), did not confirm the hypothesis that $\omega$-3PUFAs may be helpful to prevent psychosis in CHR individuals. The authors have hypothesized that such discrepancies might be explained by different overall transition rates, and by a ceiling effect due to concomitant antidepressant treatment. Other trials are currently underway to this end (Placebo-controlled Trial in Subjects at Ultra-high Risk for Psychosis With Omega-3 Fatty Acids in Europe, PURPOSE, NCT02597439). The efficacy of $\omega$-3PUFAs in subgroups of patients should also be investigated-for example, in those with aberrant membrane fatty acid levels or inflammatory markers

However, until now, recent meta-analyses have not found robust evidence to favor specific preventive interventions, as confirmed by a recent umbrella review (85), i.e. a review of seven meta-analyses in the field of preventive interventions for psychosis in CHR individuals.

Several methodological limitations of our systematic, qualitative review must be acknowledged. First, we excluded articles published in languages other than English. Second, given the relative scarcity of research on this topic to date, and the variability across studies, we were not able to conduct a quantitative systematic review or meta-analysis. Meta-analytic results would be useful to provide important information regarding common predictors and the predictive power of existing models, but these are infeasible at present given the very limited state of research in this neglected area of clinical psychiatry. Third, combining the three CHR subgroups, populations may confound predictors and have an impact on the overall conversion rates (86) and, therefore, contribute to inconsistencies across sites. As an example, if compared with individuals at genetic high risk, people with intermittent psychosis are more severely impaired and develop more frequently acute psychosis (86). Therefore, extrapolating from the whole high-risk category its three fundamental subgroups, it is likely that more accurate predictors may be detected (61)

In conclusion, our systematic review revealed that poor methods and reporting are very common in prediction of psychosis research. In line with what has been reported above, measures of discrimination and calibration of risk prediction models have been reasonable. Most of the studies are based on small samples, did not perform internal or external cross-validation, and used poor model development strategies, and this is the reason why most published models are probably overestimated and their reported predictive accuracy is likely to be overoptimistic. Therefore, the science of risk prediction models in psychiatry is at the beginning, and this is clearly evident looking at the numerous limitations that these studies revealed. However, research on validation must be done. To make these models useful in clinical practice, predictors must be easily available and assessable, and people at high risk must have access to preventive intervention, that could be considered effective and with a minimal risk of side effects. As such, further research must be conducted to create and improve efficient but also focused preventive interventions. As for psychotic disorders, research is growing up, especially toward the direction of the risk prediction and risk stratification. The research must go forward and our goal must be to make effective prediction and prevention possible.

\section{AUTHOR CONTRIBUTIONS}

$\mathrm{CM}$ and NB contributed to summarize the literature data and to write the review. NB collected literature data and organized the tables. $\mathrm{PB}, \mathrm{SB}$, and $\mathrm{PR}$ contributed to writing and supervising the review.

\section{FUNDING}

This study was supported by Ministero dell'Istruzione, dell'Università e della Ricerca-MIUR projects "Dipartimenti di Eccellenza 2018-2022" to the Department of Neuroscience "Rita Levi Montalcini.” 


\section{REFERENCES}

1. van Os J, Kapur S. Schizophrenia. Lancet (2009) 374:635-45. doi: 10.1016/ S0140-6736(09)60995-8

2. Eaton WW, Martins SS, Nestadt G, Bienvenu OJ, Clarke D, Alexandre P. The burden of mental disorders. Epidemiol Rev (2008) 30:1-14. doi: 10.1093/ epirev/mxn011

3. Yung AR, McGorry PD, McFarlane CA, Jackson HJ, Patton GC, Rakkar A. Monitoring and care of young people at incipient risk of psychosis. Schizophr Bull (1996) 22:283-303. doi: 10.1093/schbul/22.2.283

4. Riecher-Rössler A, Studerus E. High time for a paradigm shift in psychiatry. World Psychiatry (2016) 15(2):131-3. doi: 10.1002/wps.20329

5. Insel TR. Rethinking schizophrenia. Nature (2010) 468:187-93. doi: 10.1038/ nature09552

6. Fusar-Poli P, Borgwardt S, Bechdolf A, Addington J, Riecher-Rössler A, Schultze-Lutter F, et al. The psychosis high-risk state: a comprehensive stateof-the-art review. JAMA Psychiatry (2013) 70:107-20. doi: 10.1001/ jamapsychiatry.2013.269

7. Ruhrmann S, Schultze-Lutter F, Bechdolf A, Klosterkotter J. Intervention in at-risk states for developing psychosis. Eur Arch Psychiatry Clin Neurosci (2010) 260(2):S90-4. doi: 10.1007/s00406-010-0139-5

8. Bodatsch M, Ruhrmann S, Wagner M, Muller R, Schultze-Lutter F, Frommann I, et al. Prediction of psychosis by mismatch negativity. Biol Psychiatry (2011) 69(10):959-66. doi: 10.1016/j.biopsych.2010.09.057

9. Paus T, Keshavan M, Giedd JN. Why do many psychiatric disorders emerge during adolescence? Nat Rev Neurosci (2008) 9:947-57. doi: 10.1038/nrn2513

10. Kessler RC, Berglund P, Demler O, Jin R, Merikangas KR, Walters EE. Lifetime prevalence and age-of-onset distributions of DSM-IV disorders in the national comorbidity survey replication. Arch Gen Psychiatry (2005) 62:593-602. doi: 10.1001/archpsyc.62.6.593

11. Lin CH, Lane HY. Early Identification and Intervention of Schizophrenia: Insight From Hypotheses of Glutamate Dysfunction and Oxidative Stress. Front Psychiatry (2019) 27(10):93. doi: 10.3389/fpsyt.2019.00093

12. McGorry PD, Hickie IB, Yung AR, Pantelis C, Jackson HJ. Clinical staging of psychiatric disorders: a heuristic framework for choosing earlier, safer and more effective interventions. Aust N Z J Psychiatry (2006) 40:616-22. doi: 10.1080/j.1440-1614.2006.01860.x

13. McGorry PD. Issues for DSM-V: clinical staging: a heuristic pathway to valid nosology and safer, more effective treatment in psychiatry. Am J Psychiatry (2007) 164:859-60. doi: 10.1176/ajp.2007.164.6.859

14. Wigman JT, van Os J, Thiery E, Derom C, Collip D, Jacobs N, et al. Psychiatric diagnosis revisited: towards a system of staging and profiling combining nomothetic and idiographic parameters of momentary mental states. PloS One (2013) 8:e59559. doi: 10.1371/journal.pone.0059559

15. McGorry $P$, van Os J. Redeeming diagnosis in psychiatry: timing versus specificity. Lancet (2013) 381:343-5. doi: 10.1016/S0140-6736(12)61268-9

16. Hickie IB, Scott J, McGorry PD. Clinical staging for mental disorders: a new development in diagnostic practice in mental health. Med J Aust (2013) 198:461-2. doi: 10.5694/mja13.10431

17. Fusar-Poli P, Cappucciati M, Rutigliano G, Schultze-Lutter F, Bonoldi I, Borgwardt $\mathrm{S}$, et al. At risk or not at risk? A meta-analysis of the prognostic accuracy of psychometric interviews for psychosis prediction. World Psychiatry (2015) 14:322-32. doi: 10.1002/wps.20250

18. Fusar-Poli P, Schultze-Lutter F, Cappucciati M, Rutigliano G, Bonoldi I, Stahl $\mathrm{D}$, et al. The dark side of the moon: Meta-analytical impact of recruitment strategies on risk enrichment in the clinical high risk state for psychosis. Schizophr Bull (2016) 42:732-43. doi: 10.1093/schbul/sbv162

19. Pfeiffer RM, Park Y, Kreimer AR, Lacey JVJr, Pee D, Greenlee RT, et al. Risk prediction for breast, endometrial, and ovarian cancer in white women aged 50 y or older: derivation and validation from population- based cohort studies. PloS Med (2013) 10:e1001492. doi: 10.1371/journal.pmed.1001492

20. Fusar-Poli P, Bechdolf A, Taylor MJ, Bonoldi I, Carpenter WT, Yung AR, et al. At risk for schizophrenic or affective psychoses? A meta-analysis of DSM/ICD diagnostic outcomes in individuals at high clinical risk. Schizophr Bull (2013) 39:923-32. doi: 10.1093/schbul/sbs060

21. Fusar-Poli P, Tantardini M, De Simone S, Ramella-Cravaro V, Oliver D, Kingdon J, et al. Deconstructing vulnerability for psychosis: Meta-analysis of environmental risk factors for psychosis in subjects at ultra high-risk. Eur Psychiatry (2017) (40):65-75. doi: 10.1016/j.eurpsy.2016.09.003

22. Fusar-Poli P, Cappucciati M, Borgwardt S, Woods SW, Addington J, Nelson $\mathrm{B}$, et al. Heterogeneity of psychosis risk within individuals at clinical high risk: a meta-analytical stratification. JAMA Psychiatry (2016) 73:113-20. doi: 10.1001/jamapsychiatry.2015.2324

23. Ross PL, Gerigk C, Gonen M, Yossepowitch O, Cagiannos I, Sogani PC, et al. Comparisons of nomograms and urologists' predictions in prostate cancer. Semin Urol Oncol (2002) 20:82-8. doi: 10.1053/suro.2002.32490

24. Fusar-Poli P, Bonoldi I, Yung AR, Borgwardt S, Kempton MJ, Valmaggia L, et al. Predicting psychosis: meta-analysis of transition outcomes in individuals at high clinical risk. Arch Gen Psychiatry (2012) 69:220-9. doi: 10.1001/ archgenpsychiatry.2011.1472

25. Keshavan MS, Nasrallah HA, Tandon R. Schizophrenia, "Just the Facts" 6. Moving ahead with the schizophrenia concept: from the elephant to the mouse. Schizophr Res (2011) 127:3-13. doi: 10.1016/j.schres.2011.01.011

26. Fusar-Poli P, Schultze-Lutter F. Predicting the onset of psychosis in patients at clinical high risk: practical guide to probabilistic prognostic reasoning. Evidence-Based Ment Health (2016) 19:10-5. doi: 10.1136/eb-2015-102295

27. Studerus E, Ramyead A, Riecher-Rössler A. Prediction of transition to psychosis in patients with a clinical high risk for psychosis: a systematic review of methodology and reporting. Psychol Med (2017) 47(7):1163-78. doi: $10.1017 /$ S0033291716003494

28. Schmidt A, Cappucciati M, Radua J, Rutigliano G, Rocchetti M, Dell'Osso L, et al. Improving Prognostic Accuracy in Subjects at Clinical High Risk for Psychosis: Systematic Review of Predictive Models and Meta-analytical Sequential Testing Simulation. Schizophr Bull (2017) 43(2):375-88. doi: 10.1093/schbul/sbw098

29. Yung AR, Phillips LJ, McGorry PD, McFarlane CA, Francey S, Harrigan S, et al. Prediction of psychosis. A step towards indicated prevention of schizophrenia. Br J Psychiatry (1998) 172:14-20. doi: 10.1192/ S0007125000297602

30. Moher D, Liberati A, Tetzlaff J, Altman DGPRISMA Group. Preferred reporting items for systematic reviews and meta-analyses: the PRISMA statement. PloS Med (2009) 6(7):e1000097. doi: 10.1371/ journal.pmed.1000097

31. van der Ploeg T, Austin PC, Steyerberg EW. Modern modelling techniques are data hungry: a simulation study for predicting dichotomous endpoints. BMC Med Res Methodol (2014) 14:137. doi: 10.1186/1471-2288-14-137

32. Musoro JZ, Zwinderman AH, Puhan MA, ter Riet G, Geskus RB. Validation of prediction models based on lasso regression with multiply imputed data. BMC Med Res Methodol (2014) 14:116. doi: 10.1186/1471-2288-14-116

33. George B, Seals S, Aban I. Survival analysis and regression models. J Nucl Cardiol (2014) 21:686-94. doi: 10.1007/s12350-014-9908-2

34. Tuffery P, Guyon F, Derreumaux P. Improved greedy algorithm for protein structure reconstruction. J Comput Chem (2005) 26:506-13. doi: 10.1002/jcc.20181

35. Mason O, Startup M, Halpin S, Schall U, Conrad A, Carr V. Risk factors for transition to first episode psychosis among individuals with 'at-risk mental states'. Schizophr Res (2004) 71:227-37. doi: 10.1016/j.schres.2004.04.006

36. Cannon TD, Cadenhead K, Cornblatt B, Woods SW, Addington J, Walker E, et al. Prediction of psychosis in youth at high clinical risk: a multisite longitudinal study in North America. Arch Gen Psychiatry (2008) 65:2837. doi: 10.1001/archgenpsychiatry.2007.3

37. Nelson B, Yuen HP, Wood SJ, Lin A, Spiliotacopoulos D, Bruxner A, et al. Long-term follow-up of a group at ultra high risk ("prodromal") for psychosis: the PACE 400 study. JAMA Psychiatry (2013) 70:793-802. doi: 10.1001/ jamapsychiatry.2013.1270

38. Nieman DH, Velthorst E, Becker HE, de Haan L, Dingemans PM, Linszen $\mathrm{DH}$, et al. The Strauss and Carpenter Prognostic Scale in subjects clinically at high risk of psychosis. Acta Psychiatr Scand (2013) 127:53-61. doi: 10.1111/ j.1600-0447.2012.01899.x

39. Bearden CE, Wu KN, Caplan R, Cannon TD. Thought disorder and communication deviance as predictors of outcome in youth at clinical high risk for psychosis. J Am Acad Child Adolesc Psychiatry (2011) 50:669-80. doi: 10.1016/j.jaac.2011.03.021

40. DeVylder JE, Muchomba FM, Gill KE, Ben-David S, Walder DJ, Malaspina D, et al. Symptom trajectories and psychosis onset in a clinical high-risk cohort: 
the relevance of subthreshold thought disorder. Schizophr Res (2014) 159:27883. doi: $10.1016 /$ j.schres.2014.08.008

41. Ziermans T, de Wit S, Schothorst P, Sprong M, van Engeland H, Kahn R, et al. Neurocognitive and clinical predictors of long-term outcome in adolescents at ultra-high risk for psychosis: a 6-year follow-up. PLoS One (2014) 9:93994. doi: 10.1371/journal.pone.0093994

42. Riecher-Rössler A, Pflueger MO, Aston J, Borgwardt SJ, Brewer WJ, Gschwandtner U, et al. Efficacy of using cognitive status in predicting psychosis: a 7-year follow-up. Biol Psychiatry (2009) 66:1023-30. doi: 10.1016/j.biopsych.2009.07.020

43. Tarbox SI, Addington J, Cadenhead KS, Cannon TD, Cornblatt BA, Perkins DO, et al. Premorbid functional development and conversion to psychosis in clinical high-risk youths. Dev Psychopathol (2013) 25:1171-86. doi: 10.1017/ S0954579413000448

44. Ruhrmann S, Schultze-Lutter F, Salokangas RK, Heinimaa M, Linszen D, Dingemans $\mathrm{P}$, et al. Prediction of psychosis in adolescents and young adults at high risk: results from the prospective European prediction of psychosis study. Arch Gen Psychiatry (2010) 67(3):241-51. doi: 10.1001/ archgenpsychiatry.2009.206

45. Velthorst E, Nelson B, Wiltink S, de Haan L, Wood SJ, Lin A, et al. Transition to first episode psychosis in ultra high risk populations: does baseline functioning hold the key? Schizophr Res (2013) 143:132-7. doi: 10.1016/ j.schres.2012.10.025

46. van Tricht MJ, Ruhrmann S, Arns M, Müller R, Bodatsch M, Velthorst E, et al. Can quantitative EEG measures predict clinical outcome in subjects at Clinical High Risk for psychosis? A prospective multicenter study. Schizophr Res (2014) 153:42-7. doi: 10.1016/j.schres.2014.01.019

47. Perkins DO, Jeffries CD, Addington J, Addington J, Bearden CE, Cadenhead KS, et al. Towards a psychosis risk blood diagnostic for persons experiencing highrisk symptoms: preliminary results from the NAPLS project. Schizophr Bull (2015) 41:419-28. doi: 10.1093/schbul/sbu099

48. van Tricht MJ, Nieman DH, Koelman JH, van der Meer JN, Bour LJ, de Haan L, et al. Reduced parietal P300 amplitude is associated with an increased risk for a first psychotic episode. Biol Psychiatry (2010) 68:642-8. doi: 10.1016/ j.biopsych.2010.04.022

49. Ramyead A, Studerus E, Kometer M, Uttinger M, Gschwandtner U, Fuhr P, et al. Prediction of psychosis using neural oscillations and machine learning in neuroleptic-naïve at-risk patients. World J Biol Psychiatry (2016) 17:285-95. doi: 10.3109/15622975.2015.1083614

50. Koutsouleris N, Borgwardt S, Meisenzahl EM, Bottlender R, Möller HJ, Riecher-Rössler A. Disease prediction in the at-risk mental state for psychosis using neuroanatomical biomarkers: results from the FePsy study. Schizophr Bull (2012) 38(6):1234-46. doi: 10.1093/schbul/sbr145

51. Koutsouleris N, Meisenzahl EM, Davatzikos C, Bottlender R, Frodl T, Scheuerecker J, et al. Use of neuroanatomical pattern classification to identify subjects in at-risk mental states of psychosis and predict disease transition. Arch Gen Psychiatry (2009) 66(7):700-12. doi: 10.1001/ archgenpsychiatry.2009.62

52. Koutsouleris N, Riecher-Rössler A, Meisenzahl EM, Smieskova R, Studerus E, Kambeitz- Ilankovic L, et al. Detecting the psychosis prodrome across highrisk populations using neuroanatomical biomarkers. Schizophr Bull (2015) 41:471-82. doi: $10.1093 /$ schbul/sbu078

53. Hoffman RE, Woods SW, Hawkins KA, Pittman B, Tohen M, Preda A, et al. Extracting spurious messages from noise and risk of schizophrenia-spectrum disorders in a prodromal population. Br J Psychiatry (2007) 191:355- 356. doi: 10.1192/bjp.bp.106.031195

54. Koutsouleris N, Davatzikos C, Bottlender R, Patschurek-Kliche K, Scheuerecker J, Decker P, et al. Early recognition and disease prediction in the at-risk mental states for psychosis using neurocognitive pattern classification. Schizophr Bull (2012) 38:1200-15. doi: 10.1093/schbul/sbr037

55. Pukrop R, Ruhrmann S, Schultze-Lutter F, Bechdolf A, Brockhaus-Dumke A, Klosterkötter J. Neurocognitive indicators for a conversion to psychosis: comparison of patients in a potentially initial prodromal state who did or did not convert to a psychosis. Schizophr Res (2007) 92:116-25. doi: 10.1016/ j.schres.2007.01.020

56. Fusar-Poli P, Byrne M, Valmaggia L, Day F, Tabraham P, Johns L, et al. Social dysfunction predicts two years clinical outcome in people at ultra high risk for psychosis. J Psychiatr Res (2010) 44:294-301. doi: 10.1016/j.jpsychires.2009.08.016
57. Dragt S, Nieman DH, Veltman D, Becker HE, van de Fliert R, de Haan L, et al. Environmental factors and social adjustment as predictors of a first psychosis in subjects at ultra high risk. Schizophr Res (2011) 125:69-76. doi: 10.1016/ j.schres.2010.09.007

58. Buchy L, Perkins D, Woods SW, Liu L, Addington J. Impact of substance use on conversion to psychosis in youth at clinical high risk of psychosis. Schizophr Res (2014) 156:277-80. doi: 10.1016/j.schres.2014.04.021

59. Nieman DH, Ruhrmann S, Dragt S, Soen F, van Tricht MJ, Koelman JH, et al. Psychosis prediction: stratification of risk estimation with informationprocessing and premorbid functioning variables. Schizophr Bull (2014) 40:1482-90. doi: 10.1093/schbul/sbt145

60. Lencz T, Smith CW, McLaughlin D, Auther A, Nakayama E, Hovey L, et al. Generalized and specific neurocognitive deficits in prodromal schizophrenia. Biol Psychiatry (2006) 59:863-71. doi: 10.1016/j.biopsych.2005.09.005

61. Cornblatt BA, Carrión RE, Auther A, McLaughlin D, Olsen RH, John M, et al. Psychosis prevention: a modified clinical high risk perspective from the recognition and prevention (RAP) program. Am J Psychiatry (2015) 172:986-94. doi: 10.1176/appi.ajp.2015.13121686

62. Michel C, Ruhrmann S, Schimmelmann BG, Klosterkötter J, Schultze-Lutter F. A stratified model for psychosis prediction in clinical practice. Schizophr Bull (2014) 40:1533-42. doi: 10.1093/schbul/sbu025

63. Chan MK, Krebs MO, Cox D, Guest PC, Yolken RH, Rahmoune H, et al. Development of a blood-based molecular biomarker test for identification of schizophrenia before disease onset. Trans Psychiatry (2015) 5:e601. doi: 10.1038/tp.2015.91

64. Corcoran CM, Keilp JG, Kayser J, Klim C, Butler PD, Bruder GE, et al. Emotion recognition deficits as predictors of transition in individuals at clinical high risk for schizophrenia: a neurodevelopmental perspective. Psychol Med (2015) 45(14):2959-73. doi: 10.1017/S0033291715000902

65. Gschwandtner U, Pflueger MO, Semenin V, Gaggiotti M, Riecher-Rössler A, Fuhr P. EEG: a helpful tool in the prediction of psychosis. Eur Arch Psychiatry Clin Neurosci (2009) 259(5):257-62. doi: 10.1007/s00406-008-0854-3

66. Mittal VA, Walker EF, Bearden CE, Walder D, Trottman H, Daley M, et al. Markers of basal ganglia dysfunction and conversion to psychosis: neurocognitive deficits and dyskinesias in the prodromal period. Biol Psychiatry (2010) 68:93-9. doi: 10.1016/j.biopsych.2010.01.021

67. Rüsch N, Heekeren K, Theodoridou A, Muller M, Corrigan PW, Mayer B, et al. Stigma as a stressor and transition to schizophrenia after 1 year among young people at risk of psychosis. Schizophr Res (2015) 166:43-8. doi: 10.1016/j.schres.2015.05.027

68. Thompson A, Nelson B, Yung A. Predictive validity of clinical variables in the "at risk" for psychosis population: international comparison with results from the North American Prodrome Longitudinal Study. Schizophr Res (2011) 126:51-7. doi: 10.1016/j.schres.2010.09.024

69. Zimmermann R, Gschwandtner U, Wilhelm FH, Pflueger MO, RiecherRössler A, Fuhr P. EEG spectral power and negative symptoms in at-risk individuals predict transition to psychosis. Schizophr Res (2010) 123(23):208-16. doi: 10.1016/j.schres.2010.08.031

70. Yung AR, Phillips LJ, Yuen HP, McGorry PD. Risk factors for psychosis in an ultra high-risk group: psychopathology and clinical features. Schizophr Res (2004) 1 67(2-3):131-42. doi: 10.1016/S0920-9964(03)00192-0

71. Yung AR, Phillips LJ, Yuen HP, Francey SM, McFarlane CA, Hallgren M, et al. Psychosis prediction: 12-month follow up of a high-risk ("prodromal") group. Schizophr Res (2003) 60(1):21-32. doi: 10.1016/S0920-9964(02)00167-6

72. Bernardini F, Attademo L, Cleary SD, Luther C, Shim RS, Quartesan R, et al. Risk Prediction Models in Psychiatry: Toward a New Frontier for the Prevention of Mental Illnesses. J Clin Psychiatry (2017) 78(5):572-83. doi: 10.4088/JCP.15r10003

73. Steyerberg EW, Moons KG, van der Windt DA, Hayden JA, Perel P, Schroter S, et al. PROGRESS Group. Prognosis Research Strategy (PROGRESS) 3: prognostic model research. PloS Med (2013) 10(2):e1001381. doi: 10.1371/ journal.pmed.1001381

74. Mam-Lam-Fook C, Danset-Alexandre C, Pedron L, Amado I, Gaillard R, Krebs MO. Neuropsychology of subjects with ultra-high risk (UHR) of psychosis: a critical analysis of the literature. L'Encéphale (2017) 43(3):24153. doi: $10.1016 /$ j.encep.2017.02.001

75. De Herdt A, Wampers M, Vancampfort D, De Hert M, Vanhees L, Demunter $\mathrm{H}$, et al. Neurocognition in clinical high risk young adults who did or did not 
convert to a first schizophrenic psychosis: a meta-analysis. Schizophr Res (2013) 149(1- 3):48-55. doi: 10.1016/j.schres.2013.06.017

76. Moons KG, Kengne AP, Woodward M, Royston P, Vergouwe Y, Altman DG, et al. Risk prediction models: I. Development, internal validation, and assessing the incremental value of a new (bio)marker. Heart (2012) 98 (9):683-90. doi: 10.1136/heartjnl-2011-301246

77. Paulus MP. Pragmatism Instead of Mechanism: A Call for Impactful Biological Psychiatry. JAMA Psychiatry (2015) 72(7):631-2. doi: 10.1001/ jamapsychiatry.2015.0497

78. Lloyd-Jones DM. Cardiovascular risk prediction: basic concepts, current status, and future directions. Circulation (2010) 121(15):1768-77. doi: 10.1161/CIRCULATIONAHA.109.849166

79. Beswick A, Brindle P. Risk scoring in the assessment of cardiovascular risk. Curr Opin Lipidol (2006) 17(4):375-86. doi: 10.1097/01.mol.0000236362.56216.44

80. Hendriksen JM, Geersing GJ, Moons KG, de Groot JA. Diagnostic and prognostic prediction models. J Thromb Haemost (2013) 11(1):129-41. doi: $10.1111 /$ jth. 12262

81. Amminger GP, Schäfer MR, Papageorgiou K, Klier CM, Cotton SM, Harrigan $\mathrm{SM}$, et al. Long-chain omega-3 fatty acids for indicated prevention of psychotic disorders: a randomized, placebo-controlled trial. Arch Gen Psychiatry (2010) 67(2):146-54. doi: 10.1001/archgenpsychiatry.2009.192

82. Amminger GP, Schäfer MR, Schlögelhofer M, Klier CM, McGorry PD. Longerterm outcome in the prevention of psychotic disorders by the Vienna omega-3 study. Nat Commun (2015) 6:7934. doi: 10.1038/ncomms8934

83. McGorry P, Nelson B, Markulev C, Yuen. Effect of $\omega-3$ polyunsaturated fatty acids in young people at ultrahigh risk for psychotic disorders: the
NEURAPRO randomized clinical trial. JAMA Psychiatry (2017) 74(1):1927. doi: 10.1001/jamapsychiatry.2016.2902

84. Cadenhead K, Addington J, Cannon T, Cornblatt B, Mathalon D, McGlashan T. Omega-3 Fatty Acid Versus Placebo in a Clinical HighRisk Sample From the North American Prodrome Longitudinal Studies (NAPLS) Consortium. Schizophr Bull (2017) 43:S16-6. doi: 10.1093/ schbul/sbx021.042

85. Fusar-Poli P, Davies C, Solmi M, Brondino N, De Micheli A, KotlickaAntczak M, et al. Preventive Treatments for Psychosis: Umbrella Review (Just the Evidence). Front Psychiatry (2019) 11(10):764. doi: 10.3389/ fpsyt.2019.00764

86. Nelson B, Yuen K, Yung AR. Ultra high risk (UHR) for psychosis criteria: are there different levels of risk for transition to psychosis? Schizophr Res (2011) 125:62-8. doi: 10.1016/j.schres.2010.10.017

Conflict of Interest: The authors declare that the research was conducted in the absence of any commercial or financial relationships that could be construed as a potential conflict of interest.

Copyright (c) 2020 Montemagni, Bellino, Bracale, Bozzatello and Rocca. This is an open-access article distributed under the terms of the Creative Commons Attribution License (CC BY). The use, distribution or reproduction in other forums is permitted, provided the original author(s) and the copyright owner(s) are credited and that the original publication in this journal is cited, in accordance with accepted academic practice. No use, distribution or reproduction is permitted which does not comply with these terms. 